\title{
BOUNDARY-DOMAIN INTEGRAL METHOD FOR VORTICITY TRANSPORT EQUATION WITH VARIABLE VISCOSITY
}

\author{
J. RAVNIK \& J. TIBAUT \\ University of Maribor, Faculty of Mechanical Engineering, Maribor, Slovenia.
}

\begin{abstract}
In this paper, we derive a boundary-domain integral formulation for the vorticity transport equation under the assumption that the viscosity of the fluid, through which the vorticity is transported by diffusion and convection, is spatially changing. The vorticity transport equation is a second order partial differential equation of a diffusion-convection type.

The final boundary-domain integral representation of the vorticity transport equation is discretized using a domain decomposition approach, where a system of linear equations is set-up for each sub-domain, while subdomains are joint by compatibility conditions. The validity of the method is checked using several analytical examples. Convergence properties are studied yielding that the proposed discretization technique is second order accurate for constant and variable viscosity cases.

Keywords: boundary-domain element method, variable material properties, vorticity transport equation
\end{abstract}

\section{INTRODUCTION}

Convection-diffusion type partial differential equations govern the physical mechanisms of transport phenomena - transport of momentum, energy and mass. In many engineering or natural circumstances, the flow velocity or the diffusion coefficient vary with time and location. Variation may be due to the unsteady nature of the underlying phenomena or due to temperature or pressure dependence of diffusion coefficient. In terms of the boundary element method, this type of problems can in the case of the constant velocity field and constant diffusivity be described by pure boundary integral equations by using the diffusionconvection fundamental solution.

Several researchers have considered variable velocity fields. Driessen and Dohner [7] proposed finite element - boundary element method for advection-diffusion problems with variable advective fields and in finite domains. DeSilva et al. [6] considered 2D energy transport. Wrobel and DeFigueiredo [18] and Rap et al. [11] developed a dual reciprocity boundary element formulation for convection-diffusion problems with variable velocity fields.

Variable diffusivity was considered by Grzhibovskis et al. [8], Chkadua et al. [5], Al Jawary [1-3] and Ang et al. [4]. Ravnik and Skerget [12] proposed a boundary-domain integral formulation for diffusion-convection equations with variable coefficient and velocity and considered the energy equation with variable material properties, [13]. Boundary-domain integral method for compressible fluid flow was proposed by Škerget and co-workers $[15,16]$.

In this paper we, consider a spatially variable fluid viscosity and develop the boundary-domain integral method for the solution of vorticity transport equation in $3 \mathrm{D}$. The vorticity transport equation governs the advective and diffusive movement of vorticity in fluid flows. It is an inhomogeneous equation due to the presence of the vortex twisting and stretching term, which accounts for inherent the 3D nature of fluid flows. The dual reciprocity method [10], which is traditionally used to handle the domain contribution steaming from non-homogeneous parts of the governing equation, is in this work replaced by a 
domain decomposition approach, which results in sparse integral matrices and thus makes DBIM computationally affordable.

\section{GOVERNING EQUATIONS}

We consider a domain $\Omega$ with a boundary $\Gamma$ filled with an incompressible fluid. The flow of the fluid is assumed to be steady. Let $\vec{r} \in \Omega$ denote the position in the domain. If the fluid velocity field is denoted by $\vec{v}=\vec{v}(\vec{r}, t)$ then the mass conservation law for incompressible fluids states that the divergence of the velocityfield vanishes, i.e.

$$
\vec{\nabla} \cdot \vec{v}=0
$$

The steady fluid momentum transport equation for this case is

$$
(\vec{v} \cdot \vec{\nabla}) \vec{v}=-\frac{1}{\rho_{0}} \vec{\nabla} \rho+\vec{\nabla} \cdot \underline{\tau}+\vec{S}_{m},
$$

where $p$ is the pressure, $\underline{\tau}$ is the stress tensor and $\vec{S}_{m}$ accounts for any additional body forces, which may act as a sink or source of momentum.

Taking into account the Newton's law of viscosity, which is a linear relationship between stress and rate of strain, and considering incompressibility, the stress tensor may be written as $\underline{\tau}=2 \mu \underline{\dot{\epsilon}}$; where $\underline{\underline{\epsilon}}$ is the rate of strain tensor and $\mu$ is the kinematic viscosity of the fluid. The rate of strain tensor is defines as $\dot{\epsilon}_{i j}=\frac{1}{2}\left(\frac{\partial v_{i}}{\partial x_{j}}+\frac{\partial v_{j}}{\partial x_{i}}\right)$. Calculating the divergence of the stress tensor gives the following expression. $\vec{\nabla} \cdot \underline{\tau}=\mu \vec{\nabla} \cdot(2 \underline{\dot{\epsilon}})+2 \underline{\dot{\epsilon}} \cdot \vec{\nabla} \mu$ Due to incompressibility $\vec{\nabla} \cdot \vec{v}=0$, we can simplify it by showing that $\mu \vec{\nabla} \cdot(2 \underline{\dot{\epsilon}})=\mu \nabla^{2} \vec{v}$. Thus, the final expression for the momentum transport equation is

$$
(\vec{v} \cdot \vec{\nabla}) \vec{v}=-\frac{1}{\rho_{0}} \vec{\nabla} \rho+\mu \nabla^{2} \vec{v}+2 \dot{\epsilon} \cdot \vec{\nabla} \mu+\vec{S}_{m},
$$

where the fluid viscosity may vary with time and location.

To derive the velocity-vorticity formulation, we introduce vorticity, which is defined at the curl of the velocity field, $\vec{\omega}=\vec{\nabla} \times \vec{v}$. Vorticity is by definition solenoidal $\vec{\nabla} \cdot \vec{\omega}=0$. The kinematics equation is a vector elliptic partial differential equation od Poisson type, which links the velocity and vorticity fields for every point in space and time. For an incompressible fluid, it can be stated as (Ravnik [14]):

$$
\nabla^{2} \vec{v}+\vec{\nabla} \times \vec{\omega}=0
$$

The vorticity transport equation is derived by taking a curl of the momentum equations. First, the following relationships are used $(\vec{v} \cdot \vec{\nabla}) \vec{v}=\frac{1}{2} \vec{\nabla} v^{2}-\vec{v} \times \vec{\omega}, \nabla^{2} \vec{v}=-\vec{\nabla} \times \vec{\omega}$, to rewrite
the momentum equation into

$$
\frac{1}{2} \vec{\nabla} v^{2}-\vec{v} \times \vec{\omega}=-\frac{1}{\rho_{0}} \vec{\nabla} \rho-\mu \vec{\nabla} \times \vec{\omega}+2 \underline{\dot{\epsilon}} \cdot \vec{\nabla} \mu+\vec{S}_{m},
$$

Introducing the curl considering that the curl of a gradient is zero, we obtain

$$
\vec{\nabla} \times(\vec{v} \times \vec{\omega})=\vec{\nabla} \times(\mu \vec{\nabla} \times \vec{\omega})-\vec{\nabla} \times\left(2 \underline{\dot{\epsilon}} \vec{\nabla} \mu+\vec{S}_{m}\right),
$$


Next, we can use

$$
\begin{gathered}
\vec{\nabla} \times(\vec{v} \times \vec{\omega})=(\vec{\omega} \cdot \vec{\nabla}) \vec{v}-(\vec{v} \cdot \vec{\nabla}) \vec{\omega} \\
\vec{\nabla} \times(\mu \vec{\nabla} \times \vec{\omega})=\mu \vec{\nabla} \times(\vec{\nabla} \times \vec{w})-(\vec{\nabla} \cdot \vec{\omega}) \times \vec{\nabla} \mu, \\
\vec{\nabla} \times(\vec{\nabla} \times \vec{\omega})=-\nabla^{2} \vec{\omega},
\end{gathered}
$$

to write the final form of the vorticity transport equation for an incompressible fluid with variable viscosity:

$$
(\vec{v} \cdot \vec{\nabla}) \vec{\omega}=(\vec{w} \cdot \vec{\nabla}) \vec{v}+\mu \nabla^{2} \vec{\omega}+\vec{\nabla} \times \vec{S}_{m}+(\vec{\nabla} \times \vec{\omega}) \times \vec{\nabla} \mu+\vec{\nabla} \times(2 \underline{\dot{\epsilon}} \cdot \vec{\nabla} \mu) .
$$

The last two terms in equation (10) are due to variable viscosity. They vanish in the case of constant viscosity and the equation simplifies to a standard expression known for an incompressible fluid with constant material properties.

\section{BOUNDARY-DOMAIN INTEGRAL FORMULATION}

Vorticity transport equation (10) can be viewed as a Poisson type equation of the following format: $\nabla^{2} \vec{\omega}=\vec{b}$, where $\vec{b}$ represents all other terms. The boundary-domain integral formulation of a Poisson type equation is (Wrobel [17]):

$$
\begin{aligned}
\mu c(\vec{\xi}) \vec{\omega}(\vec{\xi})+\int_{\Gamma} \mu \vec{\omega} \vec{\nabla} u * \cdot \vec{n} d \Gamma & =\int_{\Gamma} \mu \vec{q} u * d \Gamma \\
& -\int_{\Omega}((\vec{v} \cdot \vec{\nabla}) \vec{\omega}-(\vec{\omega} \cdot \vec{\nabla}) \vec{v}) u * d \Omega \\
& +\int_{\Omega}((\vec{\nabla} \times \vec{w}) \times \vec{\nabla} \mu) u * d \Omega \\
& +\int_{\Omega}\left(\vec{\nabla} \times\left(2 \dot{\epsilon} \cdot \vec{\nabla} \mu+\vec{S}_{m}\right)\right) u * d \Omega
\end{aligned}
$$

where $\vec{\xi}$ is a point in the domain and $u^{*}=u^{*}(\vec{\xi}, \vec{r})=1 /(4 \pi|\vec{\xi}-\vec{r}|)$ is the fundamental solution for the diffusion operator. The last domain integral on the right hand side features a curl of body forces. This term may be reformulated using the following expression

$$
\vec{\nabla} \times\left(\left(2 \underline{\dot{\epsilon}} \cdot \vec{\nabla} \mu+\vec{S}_{m}\right) u^{*}\right)=\left(\vec{\nabla} \times\left(2 \dot{\epsilon} \cdot \vec{\nabla} \mu+\vec{S}_{m}\right)\right) u^{*}-\left(2 \underline{\dot{\epsilon}} \cdot \vec{\nabla} \mu+\vec{S}_{m}\right) \times \vec{\nabla} u *
$$

And the Gauss theorem

$$
\int_{\Omega} \vec{\nabla} \times\left(\left(2 \underline{\dot{\epsilon}} \cdot \vec{\nabla} \mu+\vec{S}_{m}\right) u^{*}\right) d \Omega=-\int_{\Gamma}\left(2 \underline{\dot{\epsilon}} \cdot \vec{\nabla} \mu+\vec{S}_{m}\right) u^{*} \times \vec{n} d \Gamma
$$

so that the calculation of the curl in the last term of equation (11) is avoided:

$$
\begin{aligned}
\mu c(\vec{\xi}) \vec{\omega}(\vec{\xi})+\int_{\Gamma} \mu \vec{\omega} \vec{\nabla} u^{*} \cdot \vec{n} d \Gamma & =\int_{\Gamma} \mu \vec{q} u^{*} d \Gamma \\
& -\int_{\Omega}((\vec{v} \cdot \vec{\nabla}) \vec{\omega}-(\vec{\omega} \cdot \vec{\nabla}) \vec{v}) u^{*} d \Omega \\
& +\int_{\Omega}((\vec{\nabla} \times \vec{\omega}) \times \vec{\nabla} \mu) u^{*} d \Omega \\
& -\int_{\Gamma}\left(2 \dot{\epsilon} \cdot \vec{\nabla} \mu+\vec{S}_{m}\right) u^{*} \times \vec{n} d \Gamma \\
& +\int_{\Omega}\left(2 \underline{\dot{\epsilon}} \cdot \vec{\nabla} \mu+\vec{S}_{m}\right) \times \vec{\nabla} u^{*} d \Omega
\end{aligned}
$$


The first domain integral on the right-hand side of equation (14) includes derivatives of the velocity and vorticity field functions. In order to avoid calculation of derivatives, we use algebraic relations to move the derivative from the unknown field function to the fundamental solution. Let us first write the first domain integral alone for $j^{\text {th }}$ component of vorticity only:

$$
\int_{\Omega}\left((\vec{v} \cdot \vec{\nabla}) \omega_{j}-(\vec{\omega} \cdot \vec{\nabla}) v_{j}\right) u^{*} d \Omega
$$

Due to the solenoidality of the velocity and vorticity fields, we may use $(\vec{w} \cdot \vec{\nabla}) v_{j}=\vec{\nabla} \cdot\left(\vec{\omega} v_{j}\right)$ and $(\vec{v} \cdot \vec{\nabla}) \omega_{j}=\vec{\nabla} \cdot\left(\vec{v} \omega_{j}\right)$ to transform equation (15) into

$$
\int_{\Omega}\left(\vec{\nabla} \cdot\left(\vec{v} \omega_{j}-\vec{\omega} v_{j}\right)\right) u * d \Omega
$$

In order to move the derivative towards the fundamental solution, the following algebraic relation $\vec{\nabla} \cdot\left(u *\left(\vec{v} \omega_{j}-\vec{\omega} v_{j}\right)\right)=u * \vec{\nabla} \cdot\left(\vec{v} \omega_{j}-\vec{\omega} v_{j}\right)+\left(\vec{v} \omega_{j}-\vec{\omega} v_{j}\right) \cdot \vec{\nabla} u *$ is used to obtain two integrals

$$
\int_{\Omega} \vec{\nabla} \cdot\left(u *\left(\vec{v} \omega_{j}-\vec{\omega} v_{j}\right)\right) d \Omega-\int_{\Omega}\left(\vec{v} \omega_{j}-\vec{\omega} v_{j}\right) \cdot \vec{\nabla} u * d \Omega
$$

The first integral may be converted to a boundary integral using a Gauss divergence theorem. Thus, the final form of the first domain integral on the right-hand side of equation (14) for $j^{\text {th }}$ vorticity component without derivatives of field functions may be stated as:

$$
\int_{\Gamma} \vec{n} \cdot\left(u *\left(\vec{v} \omega_{j}-\vec{\omega} v_{j}\right)\right) d \Gamma-\int_{\Omega}\left(\vec{v} \omega_{j}-\vec{\omega} v_{j}\right) \cdot \vec{\nabla} u * d \Omega
$$

The final integral expression for $j$-th component of vorticity is

$$
\begin{aligned}
\mu c(\vec{\xi}) \omega_{j}(\vec{\xi})+\int_{\Gamma} \mu \omega_{j} \vec{\nabla} u^{*} \cdot \vec{n} d \Gamma & =\int_{\Gamma} \mu q_{j} u * d \Gamma \\
& -\int_{\Gamma} \vec{n} \cdot\left(u *\left(\vec{v} \omega_{j}-\vec{\omega} v_{j}\right)\right) d \Gamma \\
& +\int_{\Omega}\left(\vec{v} \omega_{j}-\vec{\omega} v_{j}\right) \cdot \vec{\nabla} u^{*} d \Omega \\
& +\int_{\Omega}((\vec{\nabla} \times \vec{\omega}) \times \vec{\nabla} u)_{j} u * d \Omega \\
& -\int_{\Gamma}\left(\left(2 \dot{\epsilon} \cdot \vec{\nabla} \mu+\vec{S}_{m}\right) u^{*} \times \vec{n}\right)_{j} d \Gamma \\
& +\int_{\Omega}\left(\left(2 \dot{\epsilon} \cdot \vec{\nabla} \mu+\vec{S}_{m}\right) \times \vec{\nabla} u^{*}\right)_{j} d \Omega
\end{aligned}
$$

\section{DISCRETIZATION}

The governing equation (19) features, due to the inhomogeneous and non-linear nature of the underlying flow problem, boundary and domain integrals. Thus, we are required to discretize the boundary and the domain into boundary elements and domain cells. We choose quadrilateral boundary elements (Fig. 1) and hexahedral domain cells (Fig. 2) with quadratic interpolation for function $(u)$ and linear interpolation for flux (q). Interpolation of function and flux on each boundary element is done using a local coordinate system $(\xi, \eta)$. Node 

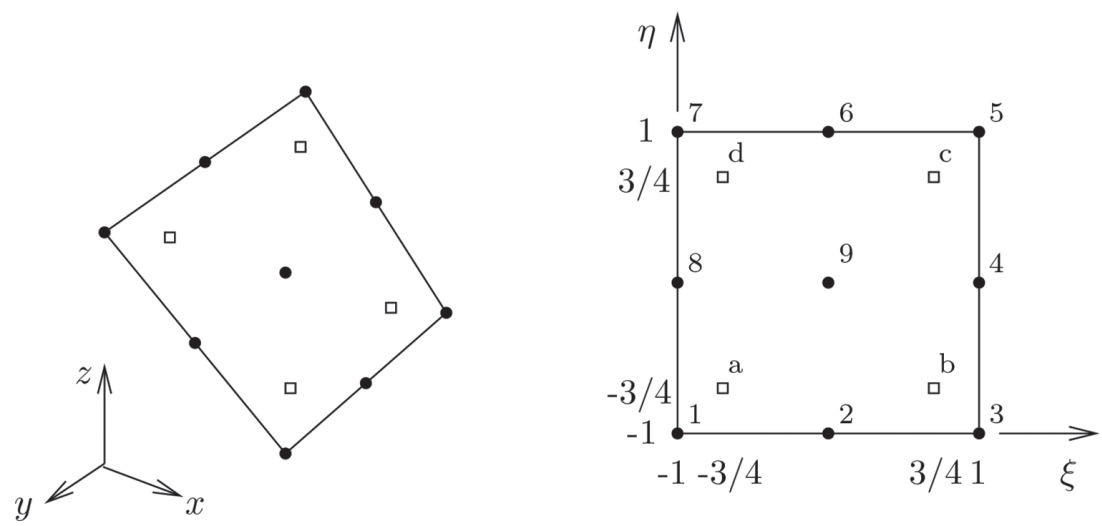

Figure 1: Linear interpolation for flux (squares) and quadratic interpolation for function (circles) over a surface. Left $\mathrm{R}^{3}$ space, right local coordinate system.

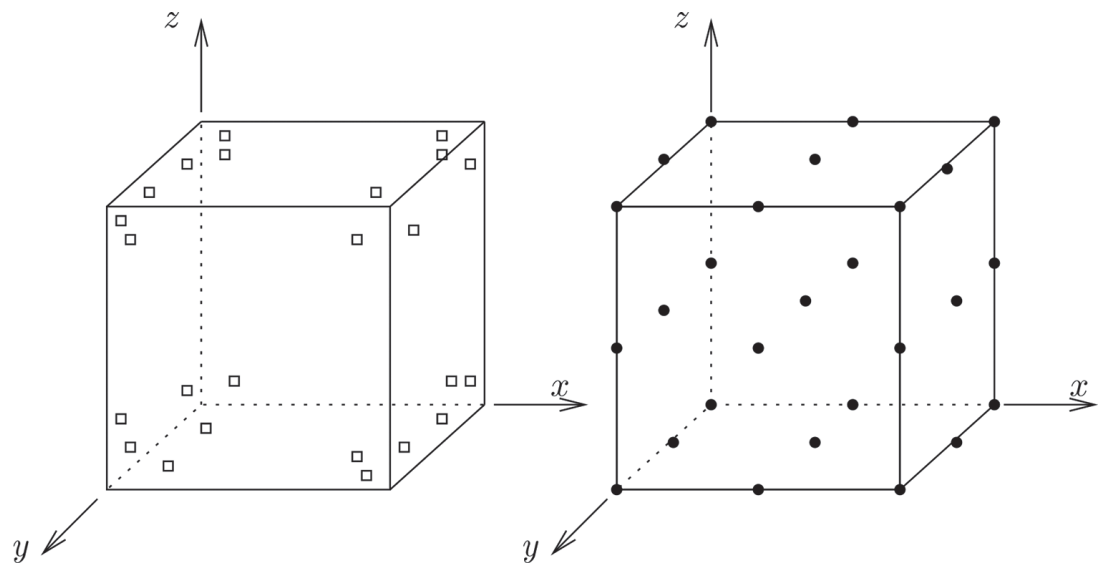

Figure 2: A computational domain consisting of one domain cell and six boundary elements. Distribution of nodes is shown: left flux, right function.

distribution is shown on Fig. 1 and the Lagrange $\varphi_{i}$ and $\phi_{i}$ shape functions are used to achieve interpolation:

$$
u(\xi, \eta)=\sum_{i=1}^{9} \varphi_{i}(\xi, \eta) u_{i} \quad q(\xi, \eta)=\sum_{i=1}^{4} \phi_{i}(\xi, \eta) q_{i}
$$

Interpolation of function within domain cells is also quadratic using 27 nodes and the Lagrange $\Phi_{i}$ shape functions are used to achieve interpolation:

$$
u(\xi, \eta, \zeta)=\sum_{i=1}^{27} \Phi_{i}(\xi, \eta, \zeta) u_{i}
$$

The geometry of the boundary element is defined by 8 corner nodes, thus each surface is defined by 4 nodes (numbers 1,3,5,7 in Fig. 1). One may find the location of flux nodes (a,b,c,d) by the following transformation 


$$
\left|\begin{array}{lll}
x_{a} & y_{a} & z_{a} \\
x_{b} & y_{b} & z_{b} \\
x_{c} & y_{c} & z_{c} \\
x_{d} & y_{d} & z_{d}
\end{array}\right|=\frac{1}{64}\left|\begin{array}{cccc}
49 & 7 & 1 & 7 \\
7 & 49 & 7 & 1 \\
1 & 7 & 49 & 7 \\
7 & 1 & 7 & 49
\end{array}\right| \cdot\left|\begin{array}{lll}
x_{1} & y_{1} & z_{1} \\
x_{3} & y_{3} & z_{3} \\
x_{5} & y_{5} & z_{5} \\
x_{7} & y_{7} & z_{7}
\end{array}\right|
$$

Based on this transformation shape functions for flux interpolation are

$$
\begin{aligned}
\phi_{1} & =\frac{4}{9}\left(\xi-\frac{3}{4}\right)\left(\eta-\frac{3}{4}\right), & \phi_{2} & =-\frac{4}{9}\left(\xi+\frac{3}{4}\right)\left(\eta-\frac{3}{4}\right) \\
\phi_{3} & =\frac{4}{9}\left(\xi+\frac{3}{4}\right)\left(\eta+\frac{3}{4}\right), & \phi_{4} & =-\frac{4}{9}\left(\xi-\frac{3}{4}\right)\left(\eta+\frac{3}{4}\right)
\end{aligned}
$$

Based on the proposed discretization scheme, in order to write the discrete version of equation (19) the following type of integrals have to be calculated

$$
\begin{gathered}
{[H]=\int_{\Gamma} \varphi_{i} \vec{\nabla} u * \cdot \vec{n} d \Gamma, \quad[G]=\int_{\Gamma} \phi_{i} u * d \Gamma,} \\
{[\vec{A}]=\int_{\Gamma} \varphi_{i} \vec{n} u * d \Gamma, \quad[\vec{D}]=\int_{\Omega} \Phi_{i} \vec{\nabla} u * d \Omega, \quad[B]=\int_{\Omega} \Phi_{i} u * d \Omega}
\end{gathered}
$$

These integrals depend on the domain geometry, computational mesh and the choice of the source point location. Due to the non-local nature of the fundamental solution, all integrals are non-zero.

Calculation of the free coefficient $c(\vec{\xi})$ When a rigid body movement is applied, $u=1$, $q=0$, we see that the sum of all $H$ matrix elements for one source point must be equal to 0 , thus we may use this fact to calculate $c(\vec{\xi})$. If the source point is located on the surface, we know that $\mathrm{c}=1 / 2$, also if the source point is inside of the element then $\mathrm{c}=1$. Those two are used to check the accuracy of calculated integrals.

\subsection{Setting up the system of equations}

We use a collocation scheme to set up linear equations at unknown nodes in the computational mesh. Since domain integrals are present in the governing integral equation this leads to full matrices of integrals and unreasonably high computational demands. To avoid this, we employ a domain-decomposition strategy to set up a sparse system of linear equations.

The strategy is as follows. We consider each domain element an individual subdomain. Such a subdomain includes 26 function nodes on the surface ( 8 in the corners, 6 at the middle of the surfaces and 12 at the middle of edges) and 24 flux nodes (4 on each side). In order to set up a system of equations, the source point is set in all of those nodes $(24+26=50)$. Additionally, the source point is set into a node in the centre of the domain element, where the function value may be obtained explicitly from known boundary values. Thus all in all, we have 51 equations for each element.

Since neighbouring elements share nodes and since boundary conditions on the outer boundaries of the domain are prescribed, we obtain an over-determined system of equations. An example of setting up the system of linear equations in this manner is shown in Fig. 3.

The system of linear equations obtain in this way is solved in a least squares manner using the Paige and Saunders [9] solver. 


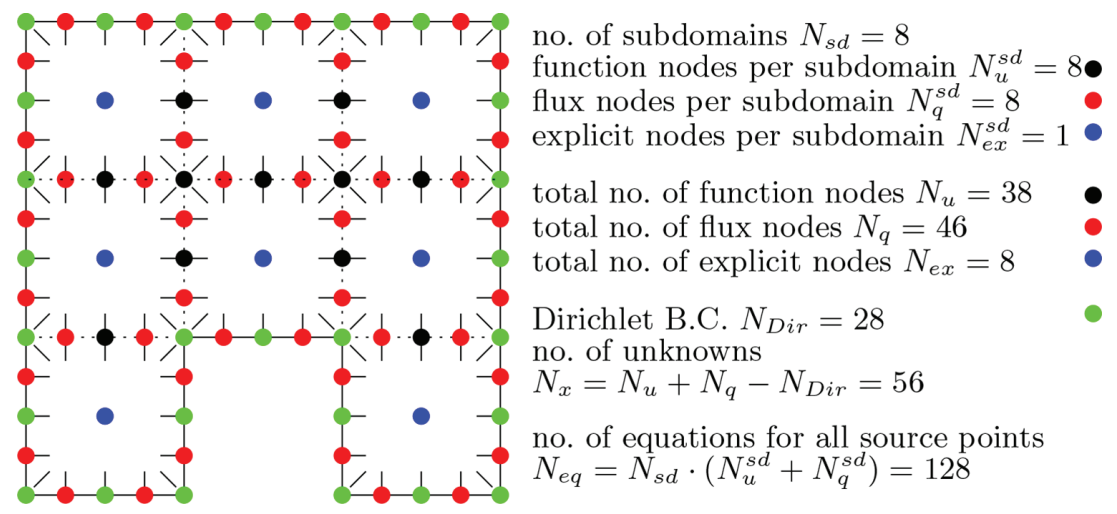

Figure 3: An example of building the system of linear equations in the proposed domaindecomposition scheme. A 2D cross-section of a domain is shown. Dirichlet boundary conditions are assumed. Each equation is shown with a short line originating from the source point location and pointing into a subdomain for each it is written.

The computational demands of the proposed scheme scale linearly with the number of nodes. In the worst case (corner node) each node is connected to eight subdomains and thus renders approximately 51.8 non-zero elements in the system matrix. If $\mathrm{n}$ is the number of all nodes, then the number of non-zero matrix elements is $51 \cdot 8 n$. For large $n$ this number of nonzero elements is significantly smaller that the $n^{2}$ storage requirements of the standard approach, which does not utilise domain decomposition.

\section{VALIDATION}

In order to validate the integral formulation of the vorticity transport equation, we solved a flow problem featuring a fluid with variable viscosity and momentum sources. We consider pressure drop driven developed flow between to large parallel horizontal plates. The vertical walls are assumed to be very far, so the flow is two-dimensional. The computational domain is shaped as a box with the flow in the $\mathrm{x}$ direction. At the inlet, a uniform flow field is prescribed $(\vec{v}=(1,0,0))$, while at the outlet a developed flow profile is prescribed $\left(d v_{x} / d_{x}=0, v_{y}=v_{z}=0\right)$. The fluid slips freely at the vertical side walls and does not slip at the top and bottom walls. We consider three cases: a constant viscosity case, a case where viscosity is a linear function of position and a case where viscosity changes quadratically. In order for the flow to have a parabolic profile in all cases, we assume that the fluid is under the influence of momentum sources. Table 1 lists all cases.

Table 1: List of cases for testing the vorticity transport equation. The analytical solution of Navier-Stokes equation for all cases is $\vec{v}-=\left(6\left(z-z^{2}\right), 0,0\right), \vec{\omega}=(0,6(1-2 z), 0)$

\begin{tabular}{lll}
\hline & Viscosity & momentum sources \\
case & $\mu$ & $\vec{S}_{m}$ \\
\hline$A$ & 1 & $(0,0,0)$ \\
$B$ & $1+z$ & $(-6(4 z-1), 0,0)$ \\
$C$ & $1+z^{2}$ & $(12(1-3 z) z, 0,0)$ \\
\hline
\end{tabular}


In order to asses the influence of the variable viscosity on the flow field results, we measure the difference between the solution obtained in the constant viscosity case $A$ and the cases with linear and quadratic dependence of viscosity. Relative RMS norm is used, defined as

$$
\left\|v_{x}\right\|=\frac{\sum_{i}\left(v_{x, A}-v_{x, B}\right)^{2}}{\sum_{i} v_{x, A}^{2}},\left\|\omega_{y}\right\|=\frac{\sum_{i}\left(\omega_{y, A}-\omega_{y, B}\right)^{2}}{\sum_{i} \omega_{y, A}^{2}}
$$

where $i$ includes all nodes along the vertical outlet pro le. We consider several meshes with equidistantly placed nodes. The meshes have 5, 9, 17, 33 and 65 nodes in each direction. For a domain of size 1 , the element sizes are between $h=0.2$ and 0.0154 .

Figure 4 displays the norms and thus compares the simulated velocity and vorticity fields for cases with and without variable viscosity. For the coarsest mesh, the difference is large, while for all others the differences are lower and at approximately the same level. Thus, we can conclude, that the proposed integral formulation and the numerical method are able to
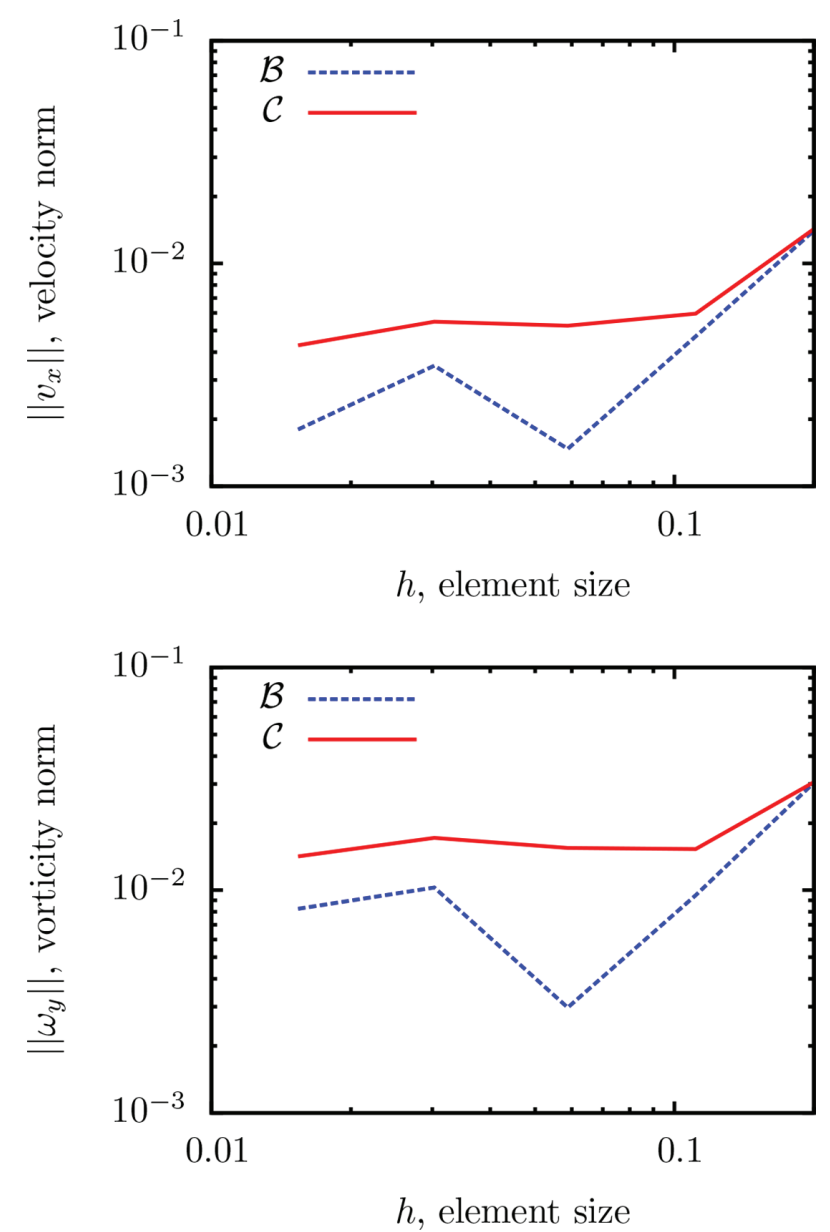

Figure 4: Velocity (top panel) and vorticity (bottom panel) norms expressing the difference between simulation of constant viscosity problem and variable viscosity problem. 
capture the physical effect of variable viscosity field in fluid flows. The norms for vorticity are higher than the norms for velocity. This is due to the fact, that vorticity is, by definition, a derivative of velocity, thus lower accuracy is expected.

\section{CONCLUSIONS}

We developed a boundary-domain integral formulation of the vorticity transport equation with variable viscosity. The contribution of the variable viscosity is present in additional integral terms which feature the viscosity gradient. These terms vanish when the viscosity is constant and the formulation simplifies to the constant viscosity case. Decomposition of the viscosity into a constant and variable part, as was proposed by other authors, was not used.

In order to efficiently employ the boundary-domain integral method on large computational grids, we propose the use of domain decomposition, which leads to over-determined systems of linear equations.

The formulation proposed in this work will be used in the future as a part of a NavierStokes fluid flow and heat transfer solver.

\section{REFERENCES}

[1] AL-Jawary, M.A., Ravnik, J., Wrobel, L.C. \& Skerget, L., Boundary element formulations for numerical solution of two-dimensional diffusion problems with variable coefficients. Computers and Mathematics with Applications, 64(8), pp. 2695-2711, 2012. https://doi.org/10.1016/j.camwa.2012.08.002

[2] AL-Jawary, M.A. \& Wrobel, L.C., Numerical solution of two-dimensional mixed problems with variable coefficients by the boundary-domain integral and integro-differential equation methods. Engineering Analysis with Boundary Elements, 35, pp. 1279-1287, 2011. https://doi.org/10.1016/j.enganabound.2011.06.004

[3] AL-Jawary, M.A. \& Wrobel, L.C., Radial integration boundary integral and integro-differential equation methods for two-dimensional heat conduction problems with variable coefficients. Engineering Analysis with Boundary Elements, 36(5), pp. 685-695, 2012. https://doi.org/10.1016/j.enganabound.2011.11.019

[4] Ang, W.T., Kusuma, J. \& Clements, D.L., A boundary element method for a second order elliptic partial differential equation with variable coefficients. Engineering Analysis with Boundary Elements, 18(4), pp. 311-316, 1996. https://doi.org/10.1016/s0955-7997(97)83178-5

[5] Chkadua, O., Mikhailov, S.E. \& Natroshvili, D., Analysis of direct boundary-domain integral equation for a mixed BVP with variable coefficient, I: equivalence and invertibility. Journal of Integral Equations and Applications, 21, pp. 499-543, 2009. https://doi.org/10.1216/jie-2009-21-4-499

[6] DeSilva, S.J., Chan, C.L., Chandra, A. \& Lim, J., Boundary element method analysis for the transient conduction convection in 2-D with spatially variable convective velocity. Applied Mathematical Modelling, 22(12), pp. 81-112, 1998. https://doi.org/10.1016/s0307-904x(98)00010-9

[7] Driessen, B.J. \& Dohner, J.L., A finite element - boundary element method for advection diffusion problems with variable advective fields and infinite domains. International Journal of Heat and Mass Transfer, 44, pp. 2183-2191, 2001.

https://doi.org/10.1016/s0017-9310(00)00226-x 
[8] Grzhibovskis, R., Mikhailov, S. \& Rjasanow, S., Numerics of boundary-domain integral and integro-differential equations for BVP with variable coefficient in 3D. Computational Mechanics, 51, pp. 495-503, 2013. https://doi.org/10.1007/s00466-012-0777-8

[9] Paige, C.C. \& Saunders, M.A., LSQR: An algorithm for sparse linear equations and sparse least squares. ACM Transactions on Mathematical Software, 8, pp. 43-71, 1982. https://doi.org/10.1145/355984.355989

[10] Partridge, P.W., Brebbia, C.A. \& Wrobel, L.C., The dual reciprocity boundary element method. Computational Mechanics Publications Southampton, U.K.; Boston : Computational Mechanics Publications; London; New York, 1992.

[11] Rap, A., Elliott, L., Ingham, D.B., Lesnic, D. \& Wen, X., DRBEM for Cauchy convection-diffusion problems with variable coefficients. Engineering Analysis with Boundary Elements, 28(11), pp. 1321-1333, 2004.

https://doi.org/10.1016/j.enganabound.2004.06.003

[12] Ravnik, J. \& Skerget, L., Integral equation formulation of an unsteady diffusion-convection equation with variable coefficient and velocity. Computers and Mathematics with Applications, 66, pp. 2477-2488, 2014. https://doi.org/10.1016/j.camwa.2013.09.021

[13] Ravnik, J., Skerget, L., Tibaut, J. \& Yeigh, B.W., Solution of energy trans-port equation with variable material properties by BEM. International Journal of Computational Methods and Experimental Measurements, 5(3), pp. 337-347, 2017. https://doi.org/10.2495/cmem-v5-n3-337-347

[14] Ravnik, J., Skerget, L. \& Zunic, Z., Velocity-vorticity formulation for 3D natural convection in an inclined enclosure by BEM. International Journal of Heat and Mass Transfer, 51(17-18), pp. 4517-4527, 2008.

https://doi.org/10.1016/j.ijheatmasstransfer.2008.01.018

[15] Skerget, L. \& Ravnik, J., BEM simulation of compressible fluid flow in an enclosure induced by thermoacoustic waves. Engineering Analysis with Boundary Elements, 33(4), pp. 561-571, 2009.

https://doi.org/10.1016/j.enganabound.2008.08.003

[16] Skerget, L. \& Samec, N., BEM for the two-dimensional plane compress-ible fluid dynamics. Engineering Analysis with Boundary Elements, 29, pp. 41-57, 2005. https://doi.org/10.1016/j.enganabound.2004.08.007

[17] Wrobel, L.C., The Boundary Element Method. John Willey \& Sons, LTD, 2002.

[18] Wrobel, L.C. \& DeFigueiredo, D.B., A dual reciprocity boundary element formulation for convection-diffusion problems with variable velocity fields. Engineering Analysis with Boundary Elements, 8, pp. 312-319, 1991.

https://doi.org/10.1016/0955-7997(91)90044-t 